\title{
Extraction par Solvant de L'or par l'Aliquat 336 Et par le TOPO Dans des Solvants Apolaires
}

\section{Laouali Ibrahim Salmana, PhD Cand.}

Laboratoire Valorisation de Matériaux, Eau et Environnement, (FAST), Université Abdou Moumouni de Niamey, Niger

Chaibou Yacouba Abdoul-Rachid, A. FAST, Département de chimie, Université d'Agadez

\section{Zanguina Adamou, Mc}

Natatou Ibrahim, Pr.

FAST, Université Abdou Moumouni de Niamey, Niger

\section{Résumé}

$\mathrm{Au}$ cours de ce travail, l'extraction liquide-liquide de l'or par un extractant neutre (TOPO) et un extractant anionique (Aliquat 336) dans une série de solvants a été étudié. L'influence du diluant, l'effet de la force ionique de la phase aqueuse et le temps d'agitation ont été également étudiés. L'or extrait a été analysé par la méthode spectrométrique UV-Visible. Les résultats de l'extraction ont montré que l'Aliquat 336 est un extractant potentiel de l'or avec un taux d'extraction de 99\%. Par contre, le TOPO a donné un taux d'extraction moyen de $45 \%$. Pour l'Aliquat 336, la désextraction en milieu aqueux a donné un faible rendement de $0,3 \%$. Le meilleur rendement a été obtenu en milieu nitrique avec un pourcentage de $99 \%$ et cela en un temps record de 2 minutes.

Mots clés : Extraction Liquide-Liquide, Aliquat 336, TOPO, Désextraction, Or 


\title{
Solvent Extraction of Gold by Aliquat 336 and TOPO in Non-Polar Solvent
}

\author{
Laouali Ibrahim Salmana, PhD Cand. \\ Laboratoire Valorisation de Matériaux, Eau et Environnement, (FAST), \\ Université Abdou Moumouni de Niamey, Niger
}

Chaibou Yacouba Abdoul-Rachid, A.

FAST, Département de chimie, Université d'Agadez

Zanguina Adamou, Mc

Natatou Ibrahim, Pr.

FAST, Université Abdou Moumouni de Niamey, Niger

\begin{abstract}
During this work, the liquid-liquid extraction of gold by neutral extractant (TOPO) and anionic extractant (Aliquat 336) in various solvents was studied. The influence of the diluent, the effect of the ionic strength of the aqueous phase and the kinetics time were also studied. The extracted gold was analyzed by the UV-Visible spectrometric method. The results of the extraction showed that Aliquat 336 is a potential gold with 99\%. On the other hand, the TOPO gave an average extraction efficiency of $45 \%$. For Aliquat 336 , the stripping in aqueous medium gave low extraction efficiency of $0.3 \%$. The best rate was obtained in a nitric medium with $99 \%$ and in record time of 2 minutes.
\end{abstract}

Keywords: Liquid-Liquid Extraction, Aliquat 336, TOPO, Stripping, Gold

\section{Introduction}

L'hydrométallurgie consiste en une succession d'opérations chimiques caractérisées par la mise en solution d'un métal et son élaboration à partir de cette solution. En ce qui concerne la séparation et la récupération des métaux, l'extraction liquide-liquide est une technique qui trouve une grande application, c'est le cas dans la chaine de traitement des minerais, ou elle est utilisée pour isoler un sel métallique de la plus part des impuretés métallique ou non, contenues dans des solutions des lixiviations (Emuri, A., et al., 2010; Abdoul-Rachid, C. Y., Salmana, L. I., Adamou, Z., Ibrahim, N., \& Ibrahim, M., 2018 ; Chaibou Yacouba, A. R., et al., 2018 ; Flett, D. S., et al., 2005). L'extraction liquide-liquide appelée aussi extraction par solvant est un procédé qui consiste à réaliser un transfert de soluté entre deux liquides non 
miscibles : l'un étant une solution aqueuse électrolytique chargée en cations métalliques et l'autre, une solution organique (solvant) composé d'un diluant non miscible à l'eau contenant en général un ou plusieurs extractants (Chaibou Yacouba, A. R., Laouali Ibrahim, S., \& Natatou, I., 2019).

L'industrie s'est très fortement investie durant de nombreuses années dans la recherche des procédés hydro-métallurgiques permettant d'envisager simultanément la séparation, la récupération et l'exploitation des produits chimiques de base avec un coût d'exploitation raisonnable (Haddaoui, J., 2004 ; Abdesselam, S., 2014). L'hydro métallurgie de l'or est basé dans la plus part des cas sur sa mise en solution par le cyanure de sodium ou de potassium La récupération de l'or est possible grâce à l'emploi de différentes techniques, comme l'extraction par solvant. Cette technique, dans le cas particulier de la récupération de l'or en milieu chlorure, a fait l'objet d'un certain nombre d'études faisant intervenir différents types d'extractants solvatants possédant des atomes d'oxygène ou de soufre, ou des extractants tels que des amines et des sels d'ammonium quaternaires (Blazy, P., El-Aid, J., 2006 ; Blazy, P., 2000, P. Diaz-Arocas, F.J. Alguacil, \& C. Caravaca, 1992).

\section{Matériel et méthodes}

\section{Matériel}

- Matériels courants du laboratoire

- Spectrophotomètre d'absorption atomique de type PERKIN ELMER

- Bruleur air/acétylène

- Lampe à cathode creuse de l'élément Au

- Lampe deutérium

\section{Réactifs}

Les produits chimiques utilisés lors de ce travail ainsi que quelques-unes de leurs propriétés physico-chimiques sont :

- Cyanure de potassium

- Eau distillée

- Solution standard de sel de l'or, le tétrachloroaurate $\left(\mathrm{AuCl}_{4}^{-}\right)$de concentration

$1000 \mathrm{mg} / \mathrm{L}$ fourni par PERKIN ELMER

- L'oxyde de tri-octyl-phosphine (TOPO) est un agent extractant solvatant ou neutre, de masse molaire 385,64 g. $\mathrm{mol}^{-1}$, de pureté supérieur à $99 \%$ et a été fourni par Merck. 


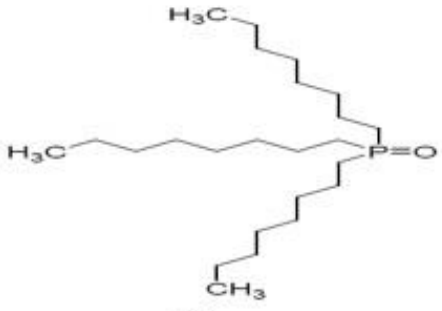

(a)

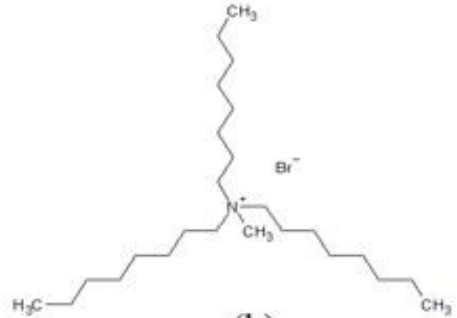

(b)

Figure 1 : Structure chimique (a) TOPO et (b) le Bromure de N-méthyl-N,N-dioctyl-1 octanaminium $\left(\mathrm{C}_{25} \mathrm{H}_{54} \mathrm{BrN}\right)$ ou Aliquat 336.

\section{Analyse par absorption atomique}

Tableau I : condition d'utilisation de l'appareil

\begin{tabular}{cc}
\hline Flamme & Air/acétylène \\
\hline Longueur d'onde & $242,8 \mathrm{~nm}$ \\
\hline Fente & $1 \mathrm{~nm}$ \\
\hline Position du bruleur & $0^{\circ}$ \\
\hline Débit de gaz air acétylène & $13,5 / 2 \mathrm{~L} / \mathrm{min}$ \\
& \\
\hline Mode de mesure & Pront \\
\hline Intensité du courant de la lampe & $4 \mathrm{~mA}$ \\
\hline Temps avant lecture & $4 \mathrm{~s}$ \\
\hline Temps de lecture & $4 \mathrm{~s}$ \\
\hline Précision & 0,2 \\
\hline Correction deuterium & Active \\
\hline Pression sortie bouteille acétylène & $0,75 \mathrm{bar}$ \\
\hline Pression compresseur air & $3,5 \mathrm{bar}$ \\
\hline
\end{tabular}

\section{Gamme d'étalonnage}

A partir d'une solution standard de l'or de concentration $1000 \mathrm{mg} / \mathrm{L}$ on opère dans des fioles jaugées de $200 \mathrm{~mL}$ quatre étalons de concentrations respectives $2,5,10$ et $20 \mathrm{mg} / \mathrm{L}$.

\section{Procédure d'extraction}

Dans des béchers de $250 \mathrm{~mL}, 10 \mathrm{~mL}$ de la phase aqueuse et $10 \mathrm{~mL}$ de la phase organique sont mises en contact et cela pendant un temps nécessaire au transfert du soluté d'une phase à l'autre jusqu'à atteindre l'équilibre d'extraction. La vitesse d'agitation a été fixée à 10 oscillations/min pendant 15 minutes sur un agitateur magnétique de marque CIMAREC. Le $\mathrm{pH}$ de la solution de l'or est de 3. A la fin de l'extraction, les deux phases sont séparées par décantation dans une ampoule à décanter. La phase aqueuse est prélevée pour déterminer la concentration en or. 
Le coefficient de distribution (1) et le pourcentage d'extraction (2) sont déterminés respectivement par les relations :

$$
\mathbf{D}=\frac{[\mathrm{M}]_{\mathrm{org}}}{[\mathrm{M}]_{\mathrm{aq}}} \quad \text { (1) } \quad \mathbf{E}(\%)=\frac{\mathbf{D}}{\mathbf{D}+\frac{\mathrm{Vaq}_{\mathrm{aq}}}{\mathrm{V}_{\text {org }}}} \times \mathbf{1 0 0}
$$

$[\mathrm{M}]_{\text {org }}=$ Concentration du métal dans la phase organique $(\mathrm{mg} / \mathrm{L})$

$[\mathrm{M}]_{\mathrm{aq}}=$ Concentration du métal dans la phase aqueuse $(\mathrm{mg} / \mathrm{L})$

\section{Résultats et Discussion}

\section{Temps d'agitation}

L'effet du temps d'agitation de l'extraction de l'or par Aliquat 336 a été étudié dans les conditions suivantes : l'extractant à $0,001 \mathrm{M}$ est dissout dans le toluène, le sel de l'or à $25 \mathrm{ppm}$ est dissout dans l'eau puis le mélange a été mis sous agitation pendant $5 \mathrm{~min}, 10 \mathrm{~min}$, jusqu'à $40 \mathrm{~min}$. les résultats sont regroupés dans la figure ci-dessous.

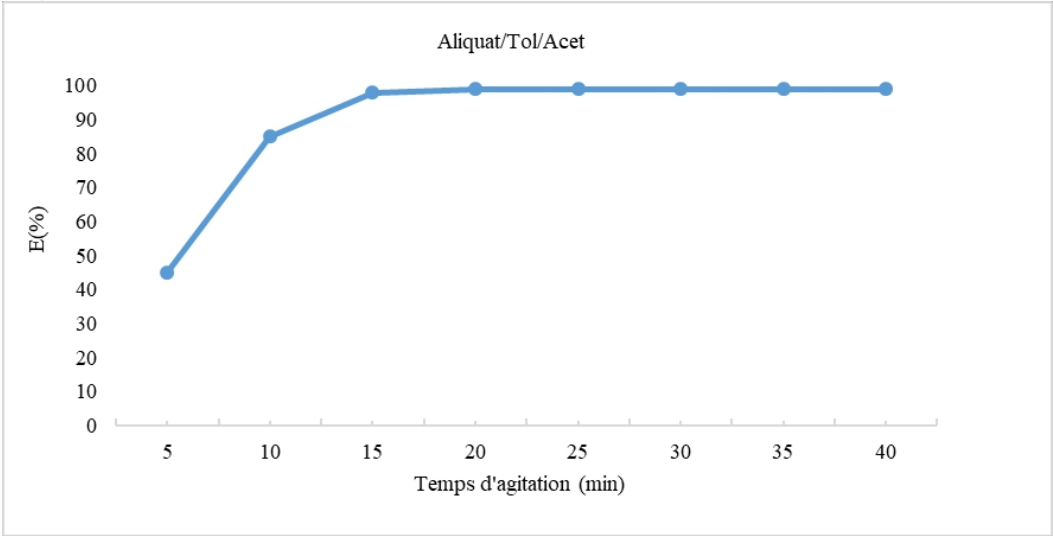

Figure 2 : Efficacité d'extraction en fonction du temps d'agitation

On remarque que le rendement d'extraction de l'or augmente considérablement en augmentant le temps d'agitation pour atteindre un maximum de $99 \%$ à partir de 15 minutes. Au-delà, le pourcentage d'extraction devient pratiquement constant. Ceci montre que l'extraction est fonction du temps d'agitation. Pour qu'une réaction soit efficace il faut un temps nécessaire de 15 minutes pour la réalisation de l'équilibre de l'extraction. Ce résultat est tout à fait comparable à d'autres travaux trouvés dans la littérature (Nassila, S., et al., 2013 ; AROUS, O., GHERROU, A., 2004 ; AROUS, O., KERDJOUDJ, H., SETA, P., 2004).

\section{Effet de la concentration}

L'extraction sel de tétrachloroaurate $\mathrm{AuCl}_{4}^{-}$à différentes concentrations (12,5 ppm, $25 \mathrm{ppm}, 50 \mathrm{ppm}, 75 \mathrm{ppm}, 100 \mathrm{ppm}$ et $125 \mathrm{ppm})$ a été effectuée avec l'Aliquat 336 et le TOPO à $0,001 \mathrm{M}$ pour la phase organique. Nous avons sur la figure ci-dessous la variation du rendement 
d'extraction de l'or dans la phase organique en fonction de la concentration du sel de l'or dans la phase aqueuse.

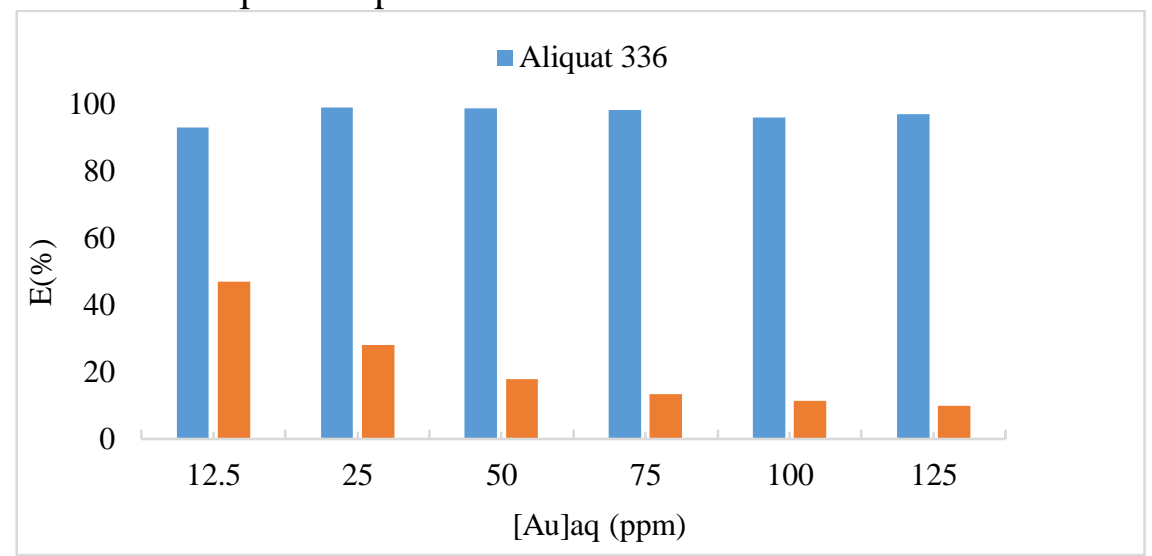

Figure 3 : Efficacité d'extraction en fonction de la nature du solvant

Nous avons étudié la capacité des ligands Aliquat 336 et TOPO à extraire de l'or à partir de la phase aqueuse. La figure 3 nous montre l'évolution de la quantité de l'or extraite en phase organique en fonction de la concentration de l'or dans la phase aqueuse (12,5 ppm à $125 \mathrm{ppm})$. Les meilleurs taux d'extractions 99\% sont obtenus avec Aliquat 336 pour une concentration en or dans la phase aqueuse de $25 \mathrm{ppm}$. Au-delà, les pourcentages de l'extraction deviennent constants. Ceci semblerait être dû à la saturation de la phase organique avec l'Aliquat 336 et par conséquent à la formation d'une couche d'émulsion qui retarde le temps de décantation. Ce qui est en accord avec les travaux des auteurs (Blazy, P., Joussemet, R., 2005 ; Diaz-Arocas, P., et al., 1993 ; Caravaca, C., et al., 1992). Par contre, avec le TOPO un taux d'extraction de $46 \%$ a été obtenu à 12,5 ppm et au-delà de cette concentration le taux d'extraction diminue jusqu'à $10 \%$ à $125 \mathrm{ppm}$. Ces résultats sembleraient être dus à la saturation des sites de complexation du TOPO.

\section{Variation du solvant}

L'efficacité des l'extractants l'Aliquat 336 et le TOPO à extraire l'or a été étudié. L'Aliquat 336 à $10^{-3} \mathrm{M}$ dans les différents diluants : tétrachlorométhane/Acétone, toluène/Acétone, diisobutylcétone/Acétone. La phase aqueuse été préparée à partir du tétrachloroaurate $\mathrm{AuCl}_{4}^{-}$à $12,5 \mathrm{ppm}$. La figure ci-dessous regroupe les pourcentages d'extraction en fonction de la nature des solvants. 


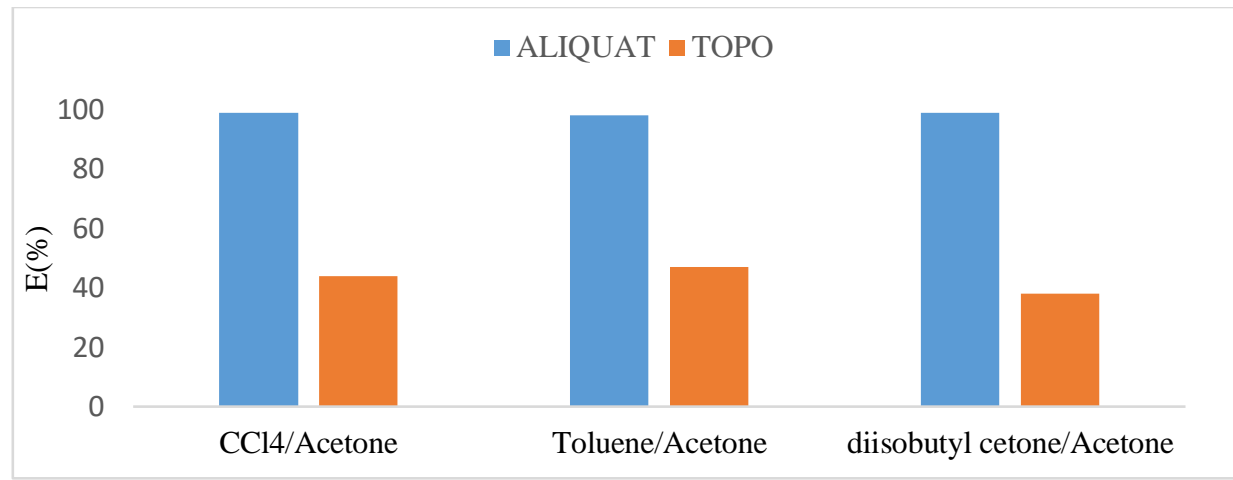

Figure 4 : Efficacité d'extraction en fonction de la nature du solvant

On remarque que pour l'Aliquat 336 et le TOPO, les rendements d'extraction sont pratiquement les même dans les solvants. Cela montre la capacité des sels ammoniums quaternaires à extraire les métaux à partir de solution aqueuse de lixiviation. L'extraction d'un métal ne pourra intervenir que dans la mesure où sa nature et celle des espèces chimiques présentes en solution aqueuse seront favorables à son engagement dans un complexe de charge négative (Paviet, P., 1992) :

Avec l'Aliquat 336.

$$
\text { On a } \quad \mathrm{M}^{\mathrm{m}+}+\mathrm{nX}^{-} \rightleftharpoons \mathrm{MX}_{\mathrm{n}}^{(\mathrm{n}-\mathrm{m})-}
$$

La réaction d'extraction s'écrit :

$$
\mathrm{MX}_{\mathrm{n}} \underset{\mathrm{aq}}{(\mathrm{n}-\mathrm{m})-}+(\mathrm{n}-\mathrm{m})\left(\mathrm{R}_{4} \mathrm{~N}^{+}, \mathrm{X}^{-}\right)_{\mathrm{org}} \underset{\mathrm{m}) \mathrm{X}_{\mathrm{aq}}^{-}}{\rightleftarrows} \mathrm{MX}_{\mathrm{n}}{ }^{(\mathrm{n}-\mathrm{m})-} \mathrm{R}_{4} \mathrm{~N}_{(\mathrm{n}-\mathrm{m})}^{+} \text {org }+(\mathrm{n}-
$$

Dans le complexe organique, le métal $\mathrm{M}$ à pour nombre de coordination : $(2 n-m)$

Les amines quaternaires représentées par 1'Aliquat 336 sont intéressantes, par leur capacité de réagir avec des solutions de forte alcalinité. Ainsi, les métaux capables de former des espèces anioniques dans ces conditions, tels que les chromates, vanadates molybdates et tungstates peuvent être extraits (Koladkar, D.V., Dhadke, P.M., 2001 ; Roundhill, D.M., 2004). Il est a remarqué dans tous les solvants le TOPO à donner un taux d'extraction moyen de $45 \%$.

\section{Extraction en présence du cyanure du potassium}

Afin d'avoir un milieu identique à celui du milieu de lixiviation de l'or avec le cyanure, nous avons étudié l'effet de l'ion cyanure sur l'extraction de l'or par Aliquat 336. L'ajout du cyanure de potassium à la phase métallique est étudié en fonction des différentes quantités en cyanure de potassium. : La phase aqueuse contient le tétracloroaurate à $25 \mathrm{ppm}$, le cyanure de potassium à $50 \mathrm{ppm}, 100 \mathrm{ppm}$ et $150 \mathrm{ppm}$. Nous avons ajouté de l'hydroxyde de 
potassium en fin de maintenir le $\mathrm{pH}$ à 11 . Trois phases organiques ont été préparées en dissolvant l'Aliquat 336 dans le toluène/acétone, tétrachlorométhane/acétone et diisobutylcetone/acétone. Les figures cidessous nous montrent le taux d'extraction obtenu avec l'Aliquat 336 dans les différents diluants.

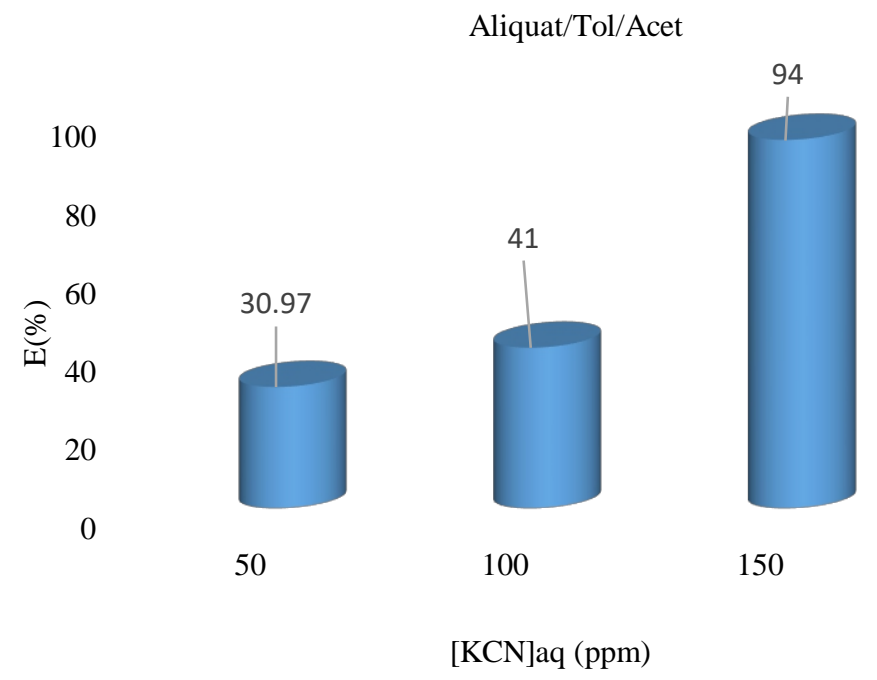

Figure 5 : Evolution de l'extraction en fonction de la variation de la concentration des sels dans la phase aqueuse

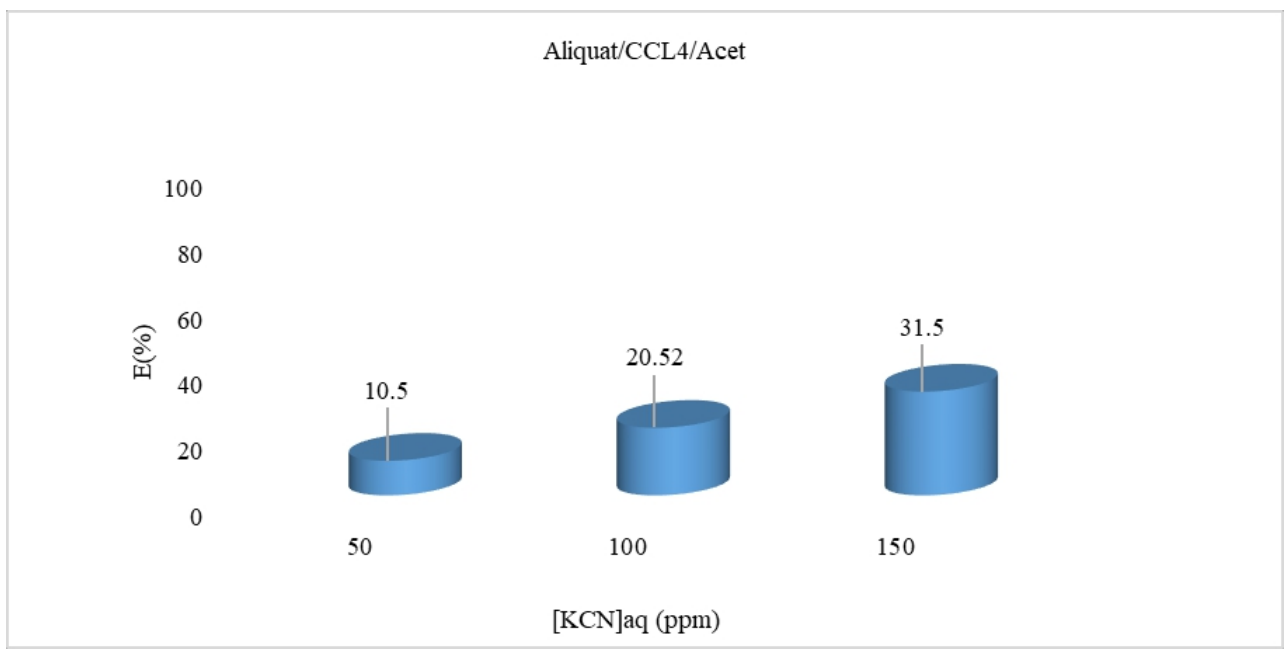

Figure 6 : Evolution de l'extraction en fonction de la variation de la concentration des sels dans la phase aqueuse 
Aliquat/Dii/Acet

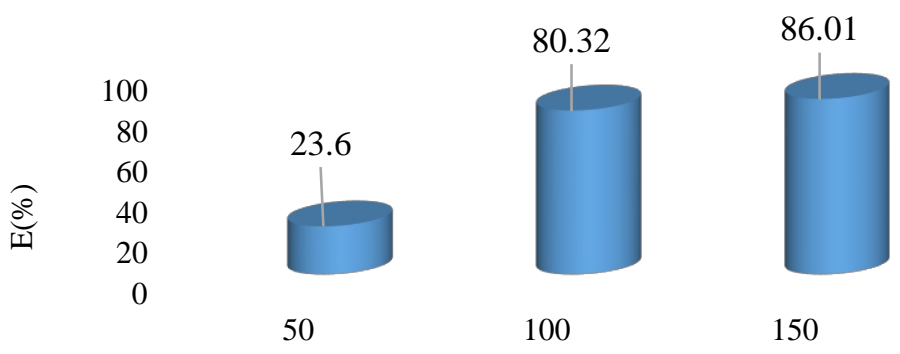

$[\mathrm{KCN}] \mathrm{aq}(\mathrm{ppm})$

Figure 7 : Evolution de l'extraction en fonction de la variation de la concentration des sels dans la phase aqueuse

L'analyse des figures 5, 6 et 7 montre que le rendement d'extraction de l'or par Aliquat 336 augmente en augmentant la concentration en cyanure de potassium dans la phase d'alimentation (la phase aqueuse). Il atteint un pourcentage de $94 \%$ dans le mélange toluène/acétone, pour des concentrations en cyanure de potassium de $150 \mathrm{ppm}$. Ce résultat montre l'effet de la concentration de cyanure de potassium sur l'extraction de l'or. Des résultats similaires ont été obtenus par (Diaz-Arocas, P., et al 1993).

\section{Effet sel de fond en variant la concentration de l'Au}

L'étude de l'extraction de l'or par Aliquat 336 a été effectuée en variant la concentration de l'or dans la phase aqueuse de $10 \mathrm{ppm}, 25 \mathrm{ppm}$ et $50 \mathrm{ppm}$. La concentration cyanure de potassium est maintenue constante à $100 \mathrm{ppm}$. La phase organique contient l'Aliquat 336 à $0,001 \mathrm{M}$ dans le toluène/acétone.

La figure 8 représente l'évolution du rendement d'extraction en fonction de la concentration de l'or.

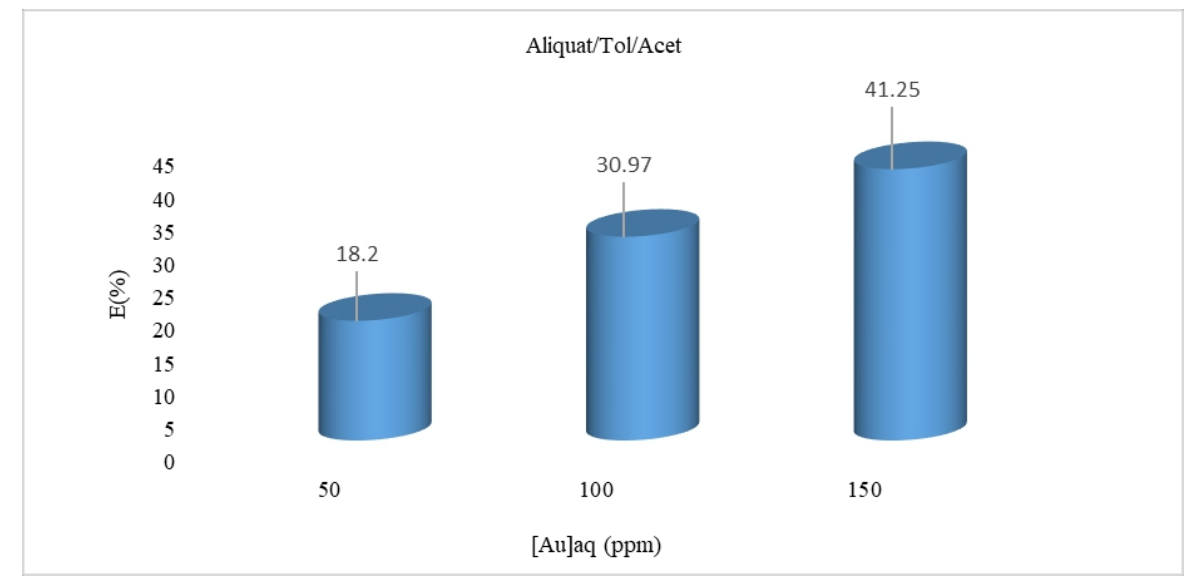

Figure 8 : Effet de la variation de la concentration de l'or 
Le résultat obtenu montre que l'extraction augmente pour atteindre un maximum de $41,25 \%$ avec une concentration de $50 \mathrm{ppm}$. Ces rendements d'extraction sont en baisse par rapport à ceux obtenu dans un milieu contenant uniquement de l'or. La diminution du rendement obtenu pouvait être due à l'extraction du potassium par l'Aliquat 336.

\section{Désextraction}

\section{Désextraction avec l'eau}

Après avoir extrait l'or avec l'Aliquat 336 avec de très bons rendements environs $99 \%$, nous avons procédé à la désextraction de ce dernier de la manière suivante : la phase organique chargée en sel de tétrachloroaurate a été mise en contact avec une nouvelle phase aqueuse contenant uniquement de l'eau. Le mélange a été agité de 5 à 40 minutes. Le tableau donne le rendement de désextraction obtenu.

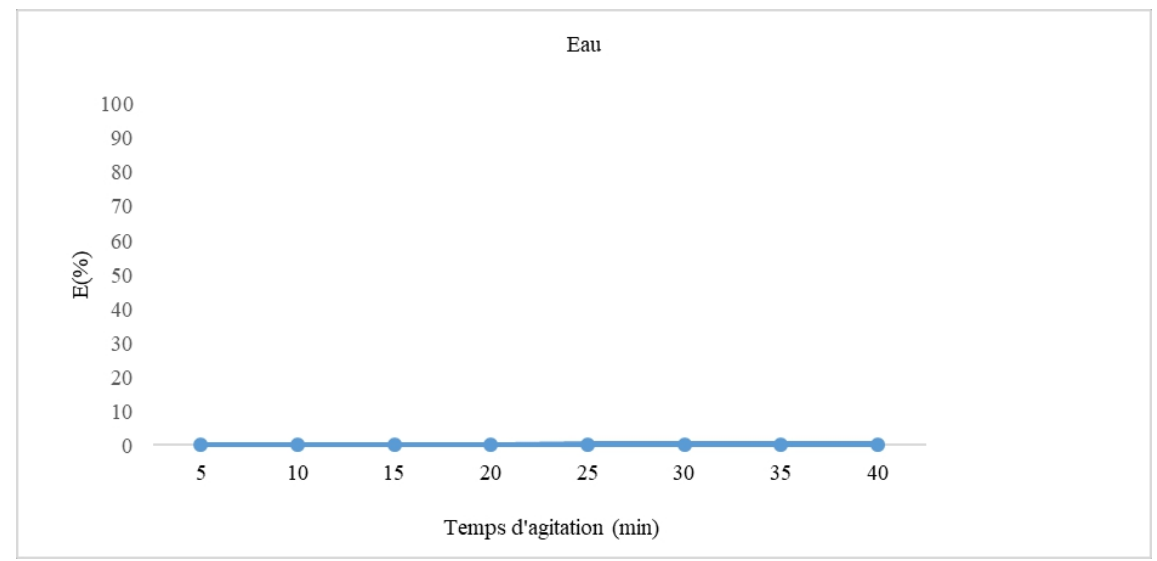

Figure 9 : Variation du temps d'agitation pour la désextraction

La figure 9 montre que la désextraction est pratiquement négligeable. Ces résultats son accord avec les travaux de (Younes, Z., 2013).

\section{Désextraction en présence des sels alcalins}

La désextraction en milieu alcalin a été étudiée dans le but de déterminer un bon rendement de désextraction de l'or par Aliquat 336. Pour cela nous avons procédé de la manière suivante : les phases organiques ont été préparées à partir des phases organiques chargées après extraction de l'or par Aliquat 336 et les phases aqueuses ont été préparées à partir des solutions de $\mathrm{KCl}$ et $\mathrm{NaOH}$. Le tableau II illustre le rendement d'extraction obtenu pour chaque cas.

Tableau II : Rendement de désextraction en milieu alcalin

\begin{tabular}{ccc}
\hline Phase aqueuse & Phase organique & Rendement $(\%)$ \\
\hline KCl $(\mathbf{0 , 5} \mathbf{M})$ & Aliquat 336/Toluène & 3,52 \\
\hline $\mathrm{NaCl}(\mathbf{0 , 5} \mathbf{M})$ & Aliquat 336/Toluène & 1,23 \\
\hline
\end{tabular}


Nous remarquons également qu'en milieu alcalin la désextraction est égalent très négligeable. Ce faible taux semblerait être du au fait que le complexe formé entre l'or et l'Aliquat 336 a une grande stabilité

\section{Désextraction en milieu acide}

\section{Avec l'acide chlorhydrique}

Toujours dans l'optique de trouver le moyen de faire la désextraction de l'or, nous avons effectué la désextraction en milieu acide. Pour cela nous avons procédé de la manière suivante : les phases organiques ont été préparées à partir des phases organiques chargées après extraction de l'or par Aliquat 336 et les phases aqueuses ont été préparées à partir des solutions d'acide chlorhydrique préparées à $0,5 \mathrm{M}$ et $1 \mathrm{M}$ pour assurer la contre extraction. Le tableau III illustre le rendement d'extraction obtenu pour chaque cas.

Tableau III: Rendement de désextraction avec l'acide chlorhydrique

\begin{tabular}{ccc}
\hline Phase aqueuse & Phase organique & Rendement (\%) \\
\hline HCl (0,5 M) & Aliquat 336/Toluène & 0,9 \\
\hline HCl (1 M) & Aliquat 336/Toluène & 16,52 \\
\hline
\end{tabular}

Contrairement aux résultats obtenus pour une désextraction avec de l'eau ou en milieu alcalin, dans ce cas une amélioration du taux désextraction est obtenue en milieu acide. Le taux de désextraction est passé à 16,52\% pour une concentration de $1 \mathrm{M}$ en acide chlorhydrique.

\section{Avec l'acide nitrique}

Pour cette partie on a utilisé de l'acide nitrique pour assurer la contre extraction, les phases organiques sont chargées à $99 \%$ en or après extraction par Aliquat 336. Le tableau IV illustre le rendement de désextraction obtenu.

Tableau IV: Rendement de désextraction avec l'acide nitrique

\begin{tabular}{ccc}
\hline Phase aqueuse & Phase organique & Rendement (\%) \\
\hline $\mathrm{HNO}_{3}(\mathbf{2 ~ M})$ & Aliquat 336/Toluène & $95 \%$ \\
\hline
\end{tabular}

Des très bons rendements sont obtenus avec l'acide nitrique. Il est à noter que la désextraction est observé directement après la mise en contact des deux phases. Une décoloration est observée ce qui montre le passage de l'or contenu dans la phase organique vers la phase aqueuse.

\section{Conclusion}

L'extraction de l'or par le TOPO et l'Aliquat 336 nous a permis de mettre au point les conditions optimales d'extraction : un temps d'agitation de 15 minutes est largement nécessaire pour effectuer une bonne extraction avec l'Aliquat 336. Ce dernier s'est révélé très sélectifs vis-à-vis de l'or avec un rendement de $99 \%$. Les pourcentages d'extraction varient entre $90 \%$ à $99 \%$ 
pour Aliquat 336 dans tous les diluants. Un taux d'extraction moyen de $45 \%$ a été obtenu avec le TOPO. Une très bonne désextraction de l'ordre de $98 \%$ a été réalisée en milieu nitrique à $2 \mathrm{M}$ et cela à un temps record.

\section{References:}

1. Abdesselam, S. (2014). Extraction liquide-liquide et Dosage spectrophotométrie duMo(IV) et du W(IV) à l'aide d'éther couronne (DB18C6). Application pour l'analyse des formes

médicamenteuses et des produits industriels, Thèse de l'Université M'Hamed Bougara-Boumerdes, Algérie.

2. Abdoul-Rachid, C. Y., Salmana, L. I., Adamou, Z., Ibrahim, N., \& Ibrahim, M. (2018). Comparative Study of Solvent Extraction of Uranium with Alamine 336 and Aliquat 336:Application to the Uranium-Bearing Solutions of Niger Republic. European Scientific Journal, ESJ, 14(9), 76. https://doi.org/10.19044/esj.2018.v14n9p76

3. Arous, O., Gherrou, A. (2004). Utilisation of mono and bicylic polyethers as mobile carriers of silver, copper and zinc through a supported liquid membrane. Sep. Sci. Technol.,39, 1681-1693.

4. Arous, O., Kerdjoudj, H., et Seta, P. (2004). Comparison of carrierfacilitated silver(I) and copper(II) ions transport mechanisms in a supported liquid membrane and in a plasticized cellulose triacetate membrane. J. Membr. Sci., 241, 177185.

5. Blazy, P., et Joussemet, R. (2005) Le traitement par gravite, Techniques de l'Ingénieur.

6. Blazy, P., et El-Aid, J. (2006). Techniques de l'Ingénieur, Métallurgie de l'or - Procédés.

7. Blazy, P. (2000). Lixiviation de thiousulfate d'or à partir d'un mécarochimique prétraite concentre de sulfure complexe.

8. Caravaca, C., Alguacil, F.J. (1992). - Hydrometal. 31. p. 257-263.

9. Chaibou Yacouba, A. R., Laouali Ibrahim, S., \& Natatou, I. (2018). Extraction liquide liquide de l'uranium et de quelques métaux restrictifs issus des jus uranifères de la République du Niger par l'acide di-2-éthylhexylphosphorique et l'oxyde de trioctylphosphine. Int. J. Biol. Chem. Sci. 12(5):2424-2437. http://ajol.info/index.php/ijbcs.

10. Chaibou Yacouba, A. R., Laouali Ibrahim, S., \& Natatou, I. (2019). Comparative study of solvent extraction of molybdenum by various extractants. Turkish Journal of Chemistry, 43(2),84-93. https://doi.org/10.3906/kim-1803-51.

11. DIAZ-Arocas, P., Alguacil, F.J., et Caravaca, C. (1993). Etude de l'extraction de l'or III en milieu chlorure par l'amine tertiaire Alamine 304. 
12. Emuri, A., Imene, R., et Mohamed, A., (2010). Extraction liquideliquide : Théorie, applications, difficultés. Ann Toxicol Anal.

13. Flett, D.S., (2005). Journal of Hazardous Materials., (120)113-118.

14. Haddaoui, J. (2004). Propriétés complexantes, extractantes et de transport descalix[4]arènes couronnes diamides en conformation cône vis-à-vis des cations alcalins. Thèse de doctorat en Physico-chimie. Université Louis Pasteur de Strasbourg. 26p. Format PDF disponible sur : http://scd-theses.u-strasbg.fr/833/01/Haddaoui2004.pdf

15. Koladkar, D.V., Dhadke, P.M. (2001). Solvent Extraction and Ion Exchange. 059-1071.

16. Nassila, S., Omar, A., and Djamel, E. A. (2013). Extraction du plomb (II) par des membranes polymères à inclusion en utilisant l'Aliquat 336 comme transporteur, Revue des sciences de l'eau Journal of Water Science.

17. Paviet, P. (1992).Application des éthers couronnes à l'extraction sélective et au dosage Technetium99, de l'iode 129 et du Césium 135 dans les effluents. Thèse de doctorat.Université Paris 6 Orsay.39p. http://www.iaea.org/inis/collection/NCLCollectionStore/Public/24/00 4/24004868.

18. Roundhill, D.M. (2004). Journal of Chemical Education. 81 (2) 275282.

19. Younes, Z. (2013). Dosage des cyanures dans les amandes amères et les noyaux d'abricot par chromatographie en phase gazeuse à détecteur ionisation de flamme en mode Head Space (HS-CPGFID) Société Française de Toxicologie Analytique https://doi:10.1051/ata/2013034. 